\title{
Can You Stand on Virtual Grounds? A Study on Postural Affordances in Virtual Reality
}

\author{
Tony Regia-Corte* \\ INRIA Rennes, France
}

\author{
Maud Marchal * \\ INSA/INRIA Rennes, France
}

\author{
Anatole Lécuyer * \\ INRIA Rennes, France
}

\begin{abstract}
The concept of affordance, introduced by the psychologist James Gibson, can be defined as the functional utility of an object, a surface or an event. The purpose of this article was to evaluate the perception of affordances in virtual environments (VE). In order to test this perception, we considered the affordances for standing on a virtual slanted surface. The participants were asked to judge whether a virtual slanted surface supported upright stance. The perception was investigated by manipulating the texture of the slanted surface (Wooden texture vs. Ice texture). Results showed an effect of the texture: the perceptual boundary (or critical angle) with the Ice texture was significantly lower than with the Wooden texture. These results reveal that perception of affordances for standing on a slanted surface in virtual reality is possible and comparable to previous studies conducted in real environments.
\end{abstract}

Index Terms: H.5.1 [nformation Interfaces and Presentation]: Multimedia Information Systems-Artificial, augmented, and virtual realities; H.1.2 [Information Systems]: User/Machine Systems-human factors; human information processing

\section{INTRODUCTION}

In daily life, we can notice that people are generally able to guide their activities in a suitable manner and without incident across the different interactions with their environment. During locomotion, for example, they succeed in both selecting optimal surfaces for supporting their weight and posture and in avoiding obstacles that could prevent locomotion. The concept of affordance, introduced by the American psychologist, James Gibson [3], defines very well this ability of the animal to guide behavior by perceiving the action possibilities offered by the surfaces of the environment. Thus, the affordance can be defined as the functional utility of an object, a surface or an event for an animal with given physical characteristics (height, weight) and some action capabilities (effectivities) defined according to the species and ontogenetic development. Similarly to the real world, virtual environments (VE) lead to a very large number of action possibilities. An important aspect regarding the interactions with VE is that these interactions involve both perception and action activities which are fundamental to affordances. Thus, the VE user has not only the possibility to perceive the VE and its different objects (via several perceptual modalities: vision, haptic, and audition) but he has also the possibility to act inside of the VE, by moving the body, walking, grasping and wielding virtual objects. These different aspects raise several important questions about the nature of VE: Are there affordances in the VE? Are we able to perceive affordances in the VE? Is there a difference between the perception of affordances in the VE and in the real world? And finally, what are the factors involved in the perception of these affordances? Therefore, the concept of affordance in the context of VE appears to be a wide and very interesting field of research

*e-mail: \{tregiaco,mmarchal,alecuyer $\}$ @ irisa.fr for both VE researchers and psychologists. However, there is very little amount of studies in which the perception of affordances has been tested in VE. Consequently, the aim of the present study is to evaluate the perception of affordances when people are inside VE. In order to test the perception of affordances in such a condition, we have chosen to consider the perception of affordances for standing on a slanted surface. This perception is basic and fundamental in the interactions with our environment. In this paper, we begin by a review on the affordances in real and virtual worlds: the concept of affordance is explained, and the previous works on the perception of affordances in the context of Virtual Reality (VR) and postural activities are described. Regarding the experiment, participants were asked to judge whether a virtual slanted surface supported upright stance. This perception was investigated by manipulating the texture of the slanted surface (Wooden texture vs. Ice texture). Finally, results were analyzed in relation to previous works and different practical implications were suggested for several domains.

\section{AfFordances in Real AND ViRTUAL WorLdS}

\subsection{The concept of Affordance}

Gibson's work, mainly centered on the field of visual perception, is at the origin of the ecological approach to perception and action as opposed to the cognitive approach found in psychology. A fundamental tenet of the ecological approach is the claim that affordances are perceived directly [3]. In other words, the perception of affordances does not require mediation or internal processing by the perceiver. The direct perception of the affordance is possible because there is invariant information in the environment that uniquely specifies that affordance. A growing body of research has demonstrated that participants are capable of perceiving affordances to control their actions in various activities including stair climbing [8, 14], sitting on surfaces [8], walking through apertures [15], and walking up slopes [5]. Although these results allow a better understanding of the perception of affordances, there is still debate between researchers whether the affordance is an inherent property of the environment or an emergent property of the animal-environment system [13]. However, in both of these theoretical views, there is an agreement on the fact that the perception of affordances involves that the environmental properties (height, width, weight, distance, etc.) are not evaluated on an extrinsic scale with the units of measurement used in physics but are measured on an intrinsic scale according to certain relevant properties of the perceiver-actor, such as its own height, width and running speed [9]. Indeed, the aforementioned studies have demonstrated that perception of affordances is based on body-scaled information. In other words, actors perceive the properties of the environment in relation to themselves. In a study of the perception of stair climbing, Warren [14] asked observers to view stairs of different heights and judge which ones they could ascend in normal fashion. Warren found that observers judgments were consistent and accurate with respect to their actual stair-climbing capabilities; each persons maximum climbable riser height was a constant proportion (.88) of leg length. Studies of other actions identified similar invariant relationships between the critical action boundary and a relevant body part across actors of different sizes: sitting [8], and passing through apertures [15]. 


\subsection{Affordances and Virtual Reality}

Several researchers consider that the Gibson's ecological framework is a promising functional approach for defining the reality of experience in relation to the problem of designing virtual environments $[2,4]$. For example, the perception of affordances could be a potential tool for sensorimotor assessment of physical presence, that is, the feeling of being physically located in a virtual place [7]. Therefore, Lepecq et al. [7] investigated the walk through a virtual aperture of variable widths. In the case of presence, the subjects body orientation, while walking, was hypothesized to be adapted to the width of the aperture and to their own shoulder width. The results of this study indicated that the locomotor postural patterns of subjects having to walk through a virtual aperture strongly resemble those of subjects who have to walk through a real aperture [15]. For most subjects, a behavioral transition from frontal walking to body rotation was observed as the width of the virtual aperture decreased. Finally, researchers have designed a conceptual model in order to evoke affordances in VE via sensory-stimuli substitution. Such a model can potentially guide VE designers in generating more ecologically valid designs [4].

\subsection{Affordances for Standing on Surfaces}

In the field of postural activities, different studies have shown that the stance can be an example of affordance; that is a given environment can afford stance for a given organism [3]. In a pioneering study, Fitzpatrick et al. [1] examined perception of affordance for supporting upright stance. The participants were asked to judge visually or haptically (i.e., by probing the surface with a hand-held rod while blindfolded) whether a slanted surface supports upright stance. In the experiments, participants stood at a distance of one meter from an inclined board, and either looked at the surface or explored the surface with the hand-held rod. Although participants were less confident and took longer to make haptic judgments in comparison to visual judgments, the perceptual boundary between supporting and not supporting did not differ for haptic and visual judgments (29.8 and 29.6 degrees respectively). The results also showed that the profiles of the responses time and confident judgments were similar for both perceptual systems: the exploration time increased and confidence decreased at the perceptual boundary. Moreover, this perceptual boundary was within a few degrees of the actual (behavioral) boundary on this behavior (approximately $30^{\circ}$ ). In a second experiment using ascending and descending methods of limits for the presentation of angles, the results also revealed that the perceptual boundaries occurred at steeper angles of inclination on descending trials than on ascending trials. This finding demonstrates a phenomenon known as enhanced contrast and suggests that perception of affordances in this task is a dynamical process [11]. In a more recent study using the same experimental paradigm, researchers have shown that the perception of affordance for supporting upright stance depended on height of center of mass [10]. In this study, participants performed the task while wearing a backpack apparatus to which masses were attached in different configurations. The developmental dimension was also examined in a study evaluating how children and adults perceived affordances for upright stance. The overall superiority of the adults relative to the children indicated clearly that there are developmental changes in the ability to perceive affordances [6]. Finally, we can notice that no studies have examined the effect of texture in this perception. This aspect will be explored in VE within the frame of the current paper.

\section{EXPERIMENT}

The aim of this experiment was to evaluate the perception of affordances in virtual reality. In order to investigate empirically this topic, we have chosen to focus our analysis on the perception of affordances for standing on a slanted surface. In this context, the per- ception was investigated by manipulating the texture of the slanted surface. To prevent an object from slipping down a slope, frictional force must be strong enough to overcome the pull of gravity. The amount of frictional force that is created depends on the coefficient of friction between the object and the surface of the slope. Thus, two contrasted textures (high-friction: Wooden vs. low-friction: Ice) were used for the slanted surface. The hypothesis of this experiment was that if the participant is able to perceive the affordance in the virtual reality, we should observe an effect of the texture: with a perceptual boundary (or critical angle) lower with the Ice texture than with the Wooden texture.

\subsection{Participants}

Twelve participants ( 2 females and 10 males) aged from 20 to 29 (mean $=24.9, \mathrm{SD}=2.8$ ), took part in this experiment. All of them were right-handed, and none of them had known perception disorders. They were all naive to the purpose of the experiment.

\subsection{Experimental Apparatus}

The experiment was conducted in a closed room with dim light. We used the eMagin Z800 Head Mounted Display as display device, at $60 \mathrm{~Hz}$ and with stereoscopy enabled. The participant was upright in front of a table with the laptop computer running the application (Figure 1) and was wearing an opaque fabric on top of the HMD to avoid seeing the surrounding real world.
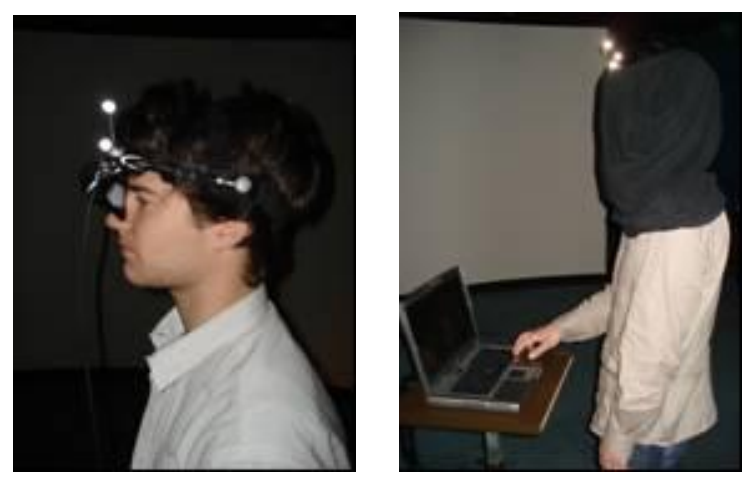

Figure 1: The experimental apparatus. Left: the participant wearing the HMD and the tracking equipment. Right: the participant equipped and wearing the opaque fabric in front of the table with the laptop computer.

The participants head was tracked by an ART ARTtrack2 infrared tracking system with 9 surrounding cameras for $360^{\circ}$ tracking. The available tracking space was a cylinder with a $3 \mathrm{~m}$ diameter and a $2.5 \mathrm{~m}$ height.

\subsection{The Virtual Environment}

In the virtual environment, the participant was inside a room (width: $8.5 \mathrm{~m} \times$ height: $4 \mathrm{~m} \times$ length: $8.5 \mathrm{~m}$ ) and stood upright $1 \mathrm{~m}$ from a slanted surface (width: $0.76 \mathrm{~m} \times$ length: $1.56 \mathrm{~m} \times$ thickness: $0.02 \mathrm{~m})$. There were no contextual cues in the room. The floor of the room was displayed with a grey carpet, the walls and the ceiling with a brown paint. Two different textures were used for the slanted surface: a Wooden texture or an Ice texture (see Figure 2). The participant controlled the inclination of the slanted surface with the keyboard of the laptop computer. The participants virtual eye height (i.e., the position of the camera) corresponded to the actual participants eye height.

\subsection{Procedure}

The task in this experiment was to adjust the angle of inclination of the virtual slanted surface until the participant felt that it was just 
barely possible for him (her) to stand on that surface with a normal upright posture. Normal upright posture was defined as standing with the feet flat (i.e., not on the toes) without bending at the hip or knees. Before the experiment, each participant was briefed about the task and was instructed to stay upright during experiment. The participant was allowed to move the head in order to explore the virtual environment. Once equipped with the HMD and the opaque fabric, the participant was conducted in front of the table with the laptop and the participants right hand was placed on the keyboard. The participant used their right hand fingers to press the computer keys. The participant could adjust the angle of the slanted surface with three keys: the up arrow to increase the inclination, the down arrow to decrease the inclination and the Enter key to validate the response. The resolution for one press on the up-down arrows was $0.25^{\circ}$ and a continuous press on the keys was possible to adjust the inclination $\left(5^{\circ} / \mathrm{sec}\right)$.
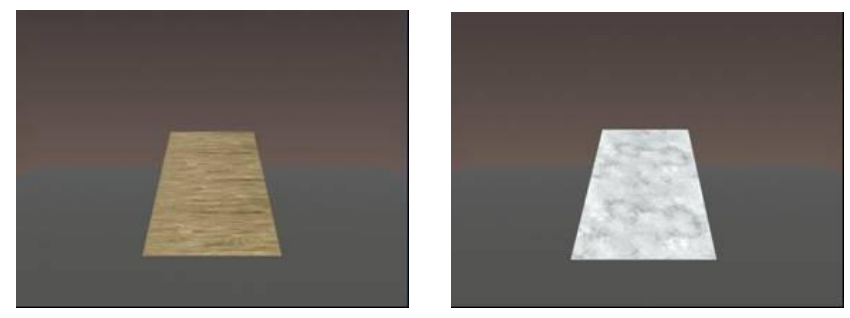

Figure 2: The participant controlled the inclination of the slanted surface with the keyboard of the laptop computer (Figure 1). The virtual environment was made up of a room with a slanted surface. Two different textures were used for the surface: a Wooden texture (left) and an Ice texture (right).

The method of adjustment was used for the measure of the critical inclination. For each trial, the angle of inclination of the surface was initially set at either the lowest angle of inclination (i.e., $0^{\circ}$ ) or the highest angle of inclination (i.e., $90^{\circ}$ ) and the participants adjusted the angle of inclination until they felt that the surface was set at the steepest angle that would support upright posture. Participants could view the surface for as long as they wished to determine whether they would be able to stand on the slanted surface. Once participants were satisfied with position of the surface, they pressed the Enter key to validate the response then a confirmation message appeared with a black screen and asked to press again the Enter key to confirm the response or to press the Space bar to return to the task. When the response was confirmed, the value of the inclination was recorded and the application displayed the next trial.

During the experiment, two different textures were used for the slanted surface: a Wooden texture and an Ice texture. No information was communicated to the participant about the texture of the slanted surface. Participants completed all the two texture conditions (Wooden and Ice) and the order of the conditions was counterbalanced across participants. In each condition, participants completed two ascending trials (in which the angle of inclination was initially set at $0^{\circ}$ ) and two descending trials (in which the angle of inclination was initially set at $90^{\circ}$ ). Ascending and descending trials alternated within a given condition, and the order of the sequence (i.e., whether an ascending or a descending trial was presented first in a given condition) was counterbalanced across participants. In this experiment, participants completed a total of 8 trials ( 2 texture conditions $\times 2$ directions $\times 2$ trials per condition). The duration of the experiment was approximatively 10 minutes.

\subsection{Results}

The mean angle of inclination chosen by the participants was considered as the perceptual boundary. A 2 (Texture: wooden vs. ice) $\times 2$ (Direction: ascending vs. descending) repeatedmeasures ANOVA was conducted on these perceptual boundaries. The ANOVA revealed a significant effect of texture, $F(1,11)=8.07$, $\mathrm{p}=0.016$ (see Figure 3 ), the perceptual boundary with the Ice texture $\left(\mathrm{M}=22.13^{\circ}, \mathrm{SD}=8.52^{\circ}\right)$ was significantly lower than with the Wooden texture $\left(\mathrm{M}=27.60^{\circ}, \mathrm{SD}=10.57^{\circ}\right)$.

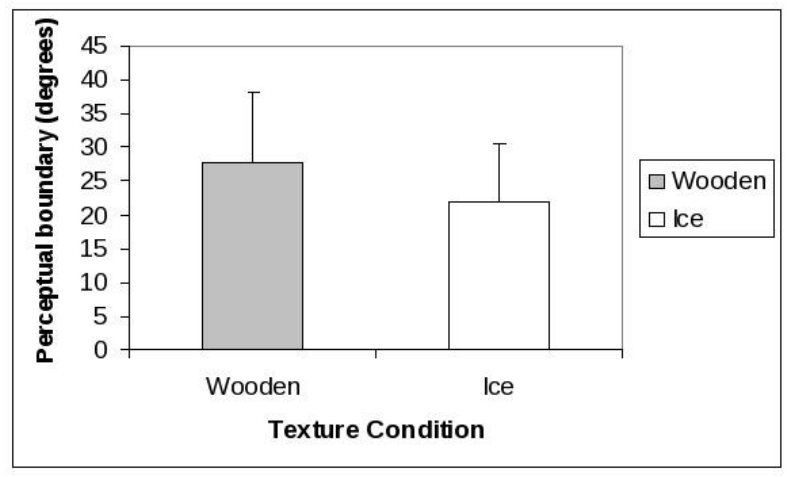

Figure 3: Perceptual boundary (or critical angle in degrees for standing on the slanted surface) as a function of the texture condition (Wooden and Ice).

The ANOVA also revealed a significant effect of direction, $\mathrm{F}(1,11)=6.83, \mathrm{p}=0.024$, the perceptual boundary occurred at a steeper angle of inclination when the surface was descending (M $\left.=26.09^{\circ}, \mathrm{SD}=9.89^{\circ}\right)$, than when the surface was ascending $(\mathrm{M}=$ $23.65^{\circ}, \mathrm{SD}=8.34^{\circ}$ ). The interaction between texture and direction was not significant $(\mathrm{F}(1,11)=1.38, \mathrm{p}=0.26)$.

\section{Discussion}

This paper analyzed the perception of affordances for standing on a slanted surface in VR. During the experiment, participants were asked to judge whether a virtual slanted surface supported upright stance. Interestingly, participants showed a natural ability to perceive affordances in VR although they have no prior experience with the virtual slanted surface displayed. These results are interesting because they are consistent with the previous research conducted in real environments but also because they reveal several specificities. In our experiment, the texture of the slanted surface was manipulated. Results showed that the perceptual boundary with the Ice texture $\left(22.13^{\circ}\right)$ was significantly lower than with the Wooden texture $\left(27.60^{\circ}\right)$. This result revealed that the virtual information about friction was detected and used in VE. Thus, participants were able to differentiate visually a low-friction texture (Ice) from a high-friction texture (Wooden). Regarding the values of perceptual boundaries observed in our experiment, the comparison with previous works revealed that the perceptual boundary with the Wooden texture $\left(27.60^{\circ}\right)$ was close to those observed in real environments $\left(29.25^{\circ}, 31.40^{\circ}\right.$ and $\left.31.13^{\circ}\right)$ with a slight underestimation. A comparison of the values obtained both in real and virtual environments is provided in Table 1.

Furthermore, as in the previous works, our results also revealed that the perceptual boundaries occurred at steeper angles of inclination on descending trials than on ascending trials. This finding demonstrates a phenomenon known as enhanced contrast [11] and suggests that perception of affordances in this task is a dynamical process. This last result reinforces again the similarity observed between the perception in VE and real environments.

The slight underestimation observed between the perceptual boundaries in our experiment and previous works conducted in real environments is an interesting and paradoxal result. Indeed, we can imagine that people inside VE are aware to be in an unrealistic 


\begin{tabular}{|c|c|c|c|c|}
\cline { 3 - 5 } \multicolumn{2}{c|}{} & \multicolumn{3}{c|}{ Perceptual boundaries (in degrees) } \\
\cline { 3 - 5 } \multicolumn{1}{c|}{$\begin{array}{c}\text { Virtual } \\
\text { Env. }\end{array}$} & Our study & $27.60^{\circ}$ & $26.16^{\circ}$ & $29.04^{\circ}$ \\
\hline \multirow{4}{*}{$\begin{array}{c}\text { Real } \\
\text { Env. }\end{array}$} & $\begin{array}{c}\text { Fitzpatrick } \\
\text { et al. [1] }\end{array}$ & $29.25^{\circ}$ & $27.25^{\circ}$ & $30.45^{\circ}$ \\
\cline { 2 - 5 } & $\begin{array}{c}\text { Klevberg and } \\
\text { Anderson [6] }\end{array}$ & $31.40^{\circ}$ & - & - \\
\cline { 2 - 5 } & $\begin{array}{c}\text { Regia-Corte and } \\
\text { Wagman [10] }\end{array}$ & $31.13^{\circ}$ & $28.71^{\circ}$ & $33.56^{\circ}$ \\
\hline
\end{tabular}

Table 1: Perceptual boundaries (or critical angle in degrees for standing on the slanted surface) for a Wooden texture as a function of the studies (in Virtual and Real environments). Mean, ascending and descending boundaries are distinguished.

world where their physical intregrity is not involved and where it is possible to risk dangerous behaviors. However, this slight underestimation indicated, on the contrary, that participants were more careful in VE. One possible explanation for this paradoxal result would be the presence of a time effect on the perception: at the beginning, the VE as a new environment involves a safety first effect with an underestimation of action possibilities. But during time and practice inside the VE, participants become more adapted and confident with a virtual perception reaching the real perception. And finally, participants adopt risky and dangerous behaviors leading to an overestimation of action possibilities. Thus, it would be interesting for the future research to consider the time factor in order to test this hypothesis.

It is important to notice that previous studies have shown that distances appear to be compressed in immersive virtual environments presented via head mounted display systems, relative to in the real world [12]. Thus, the slight underestimation observed in our study could indicate that the perception of affordances in VR would be also affected by the effect of compression. Research on this topic would be very interesting.

Regarding the practical implications of our study, the results suggest that people with motor impairments or balance disorders might improve their postural ability with specific activities in VR where they are confronted to different affordances. On the other hand, other practical implications would be in the context of urban projects, where the immersion in the $3 \mathrm{D}$ representations of buildings would allow to locate the uncomfortable affordances. The results observed in the present work call for additional investigations devoted to evaluate the perception of different affordances in VR (walking up slopes, stair climbing, gap crossing, and object reaching). It would be also interesting to conduct these investigations by considering different perceptual modalities (vision, haptic and audition).

\section{CONCLUSION}

The purpose of this work was to evaluate the perception of affordances in virtual reality taking as an example standing on a slanted surface. During the experiment, participants were asked to judge whether a virtual slanted surface supports upright stance. The task was to adjust the angle of inclination of the virtual slanted surface until the participant felt that it was just barely possible for him (her) to stand on that surface with a normal upright posture. The perception was investigated by manipulating the texture of the slanted surface. Thus, two contrasted textures (high-friction: Wooden vs. low-friction: Ice) were used for the slanted surface. Results showed an effect of the texture: the perceptual boundary (or critical angle) with the Ice texture was significantly lower than with the Wooden texture. Such results indicate that the perception of affordances for standing on a slanted surface in virtual reality is possible and comparable (with a slight underestimation) to previous studies conducted in real environments. Interestingly, it appears that virtual information about friction can be detected and used in VE. Finally, future research could investigate more precisely the role of texture and other factors on this perception in both virtual and real environments. Furthermore, future work could also evaluate the effects of order and transfer between real and virtual environments.

\section{ACKNOWLEDGEMENTS}

The authors would like to thank Mr. Laurent Bonnet and Mr. Gabriel Cirio for their help on the design of the virtual reality setup and experimental benchmark. This work was supported by the European community under FP7 FET- Open grant agreement $\mathrm{n}^{\circ} 222107$ NIW Natural Interactive Walking.

\section{REFERENCES}

[1] P. Fitzpatrick, C. Carello, R. C. Schmidt, and D. Corey. Haptic and visual perception of an affordance for upright posture. Ecological Psychology, 6(4):265-287, 1994.

[2] J. Flash and J. Holden. The reality of experience: Gibsons way. Presence: Teleoperators and Virtual Environnements, 7(1):90-95, 1998

[3] J. Gibson. The Ecological Approach to visual perception. Hillsdale, NJ: Lawrence Erlbaum Associates, Inc, original work published in 1979 edition, 1986.

[4] D. Gross, K. Stanney, and L. Cohn. Evoking affordances in virtual environments via sensori-stimuli substitution. Presence: Teleoperators and Virtual Environnements, 14(4):482-491, 2005.

[5] J. Kinsella-Shaw, B. Shaw, and M. Turvey. Perceiving walk-on-able slopes. Ecological Psychology, 4(4):223-239, 1992.

[6] G. Klevberg and D. Anderson. Visual and haptic perception of postural affordances in children and adults. Human Movement Science, 21(2):169-186, 2002.

[7] J.-C. Lepecq, L. Bringoux, J.-M. Pergandi, T. Coyle, and D. Mestre. Afforded actions as a behavioral assessment of physical presence in virtual environments. Virtual Reality, 13(3):141-151, 2009.

[8] L. Mark. Eyeheight-scaled information about affordances: A study of sitting and stair climbing. Journal of Experimental Psychology: Human Perception and Performance, 13(3):361-370, 1987.

[9] R. Oudejans, C. Michaels, F. Bakker, and M. Dolne. The relevance of action in perceiving affordances: Perception of catchableness of fly balls. Journal of Experimental Psychology: Human Perception and Performance, 22(4):879-891, 1996.

[10] T. Regia-Corte and J. Wagman. Perception of affordances for standing on an inclined surface depends on height of center of mass. Experimental Brain Research, 191(1):25-35, 2008.

[11] M. Richardson, K. Marsh, and R. Baron. Judging and actualizing intrapersonal and interpersonal affordances. Journal of Experimental Psychology: Human Perception and Performance, 33(4):845-859, 2007.

[12] F. Steinicke, G. Bruder, K. Hinrichs, M. Lappe, B. Ries, and V. Interrante. Transitional environments enhance distance perception in immersive virtual reality systems. In Proceedings of the 6th Symposium on Applied Perception in Graphics and Visualization, pages 19-26, 2009.

[13] T. Stoffregen. Affordances as properties of the animal-environment system. Ecological Psychology, 15(2):115-134, 2003.

[14] W. Warren. Perceiving affordances: Visual guidance of stair climbing. Journal of Experimental Psychology: Human Perception and Performance, 10(5):683-703, 1984

[15] W. Warren and S. Whang. Visual guidance of walking through apertures: Body-scaled information for affordances. Journal of Experimental Psychology: Human Perception and Performance, 13(3):371$383,1987$. 